\title{
Vinylketene aus Cyclobutenonen durch elektrocyclische Ringöffnung
}

Von Herbert Mayr ${ }^{[*]}$

Das optisch aktive 2,4-Dichlor-3-phenyl-cyclobutenon racemisiert über das offenkettige Vinylketen, das in geringer Konzentration im Gleichgewicht vorliegt ${ }^{11}$. Für die thermische Bildung von $\alpha$-Naphtholen aus Diphenylketen und Alkinen wurden Cyclobutenone und Vinylketene schon 1941 als Zwischenstufen vermutet ${ }^{[2]}$; die Cyclobutenone wurden später nachgewiesen ${ }^{(3)}$. Wir berichten über die elektrocyclische Ringöffnung der leicht zugänglichen Cyclobutenone ${ }^{i 4 !}$.

[*] Dr. H. Mayr

Institut für Organische Chemie der Universitä

8 München 2, Karlstraße 23 


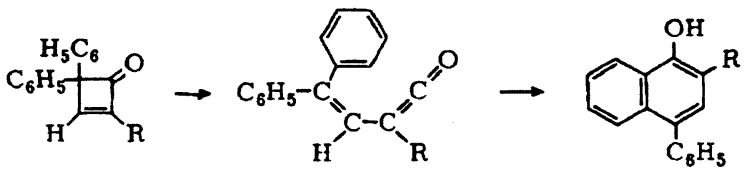

(I), $\mathrm{R}=\mathrm{H}$

(4)

(2), $\mathrm{R}=\mathrm{CH}_{3}$

(5), $\mathrm{R}=\mathrm{H}$

(6), $\mathrm{R}=\mathrm{CH}_{3}$

(3), $\mathrm{R}=\mathrm{C}_{2} \mathrm{H}_{5}$

(7), $\mathrm{R}=\mathrm{C}_{2} \mathrm{H}_{5}$

Die 4,4-Diphenyl-cyclobutenone (1)-(3) gingen beim Kochen $(1 \mathrm{~h})$ in Cyclohexan quantitativ in die $\alpha$-Naphthole $(5)^{(5)}$ bis (7) über. DaB in Anwesenheit von Alkoholen neben den Naphtholen auch $\beta, \gamma$-ungesättigte Carbonsäureester auftraten, ohne daß die Brutto-Geschwindigkeitskonstante zunahm ${ }^{[6]}$, läßt die Vinylketene (4) als logische Zwischenstufe erscheinen. Der $\alpha$-Naphthol-Bildung liegt eine intramolekulare, elektrophile Kernsubstitution zugrunde, die so rasch ist, daß die gelbe Farbe von (4) nicht in Erscheinung tritt.<smiles>CCCCCC=C(C)C(=O)C=C(C)C=C(C)C</smiles>

(8) (9)

(10)

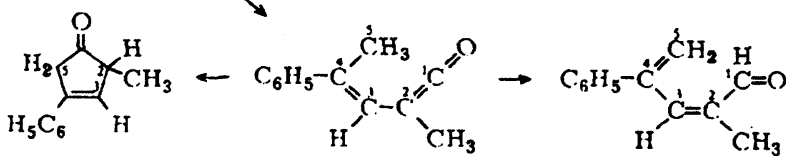

(II)

(12)

Das Cyclobutenon (8) erbrachte nach $24 \mathrm{~h}$ in siedendem Cyclohexan 64\% eines Gemischs aus 49\% (10), 21\% (13) und $30 \%$ (11). Von den cis,trans-isomeren Vinylketenen (9) und (12) bietet nur (9) die strukturellen Voraussetzungen für den Naphthol-Ringschluß zu (10). (12) trat in höherer Stationärkonzentration auf als (9) und verriet sich durch die gelbe Farbe, die auf Alkoholzusatz hin sogleich verschwand. Bei einem mobilen Gleichgewicht $(8) \rightleftharpoons(12)$ wäre eine rasche Abreaktion über (9) zu erwarten.

Die Struktur des Pentadienal-Derivats (13) ergab sich aus der CO-Bande bei $1684 \mathrm{~cm}^{-1}$ und dem ${ }^{1} \mathrm{H}-\mathrm{NMR}$-Spektrum $\left(\mathrm{CDCl}_{3}\right)$, das bei $\tau=0.51$ das Aldehydproton zeigt. Die beiden $5-\mathrm{H}$ bilden ein AB-Spektrum bei $\tau=4.26$ und $4.58 \mathrm{mit}$ $\mathrm{J}=1.5 \mathrm{~Hz}$; das Triplett bei 4.58 weist auf eine zusätzliche, gleich große Kopplung mit 3-H. Auch die Allylkopplung des $2-\mathrm{CH}_{3}$ (d bei 8.31 ) beträgt $1.5 \mathrm{~Hz}$, so $\mathrm{daB} 3-\mathrm{H}$ als Quintuplett bei 3.00 erscheint. Die Lichtabsorption des tiefroten 2,4-Dinitrophenylhydrazons von (I3) $\left(\lambda_{\max }=3.82 \mathrm{~nm}, \log \varepsilon=4.53\right.$, Äthanol) entsprach der Erwartung.

Die IR-Absorption von (11) bei $1733 \mathrm{~cm}^{-1}$ deutete auf ein nichtkonjugiertes Keton, desgleichen die Spektren des in<smiles>CC(C)=CC(=O)C(C)C</smiles>

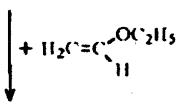

(16)

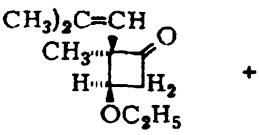

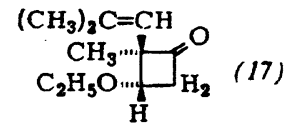

Acetat-Puffer bereiteten Semicarbazons. Das 2.4-Dinitrophenylhydrazon (violettrote Nadeln, mit schwefelsaurem 2,4-Dinitrophenylhydrazin erhalten) ist dagegen von einem isomeren, konjugierten Keton abgeleitet. Authentisches 5-Methyl-3-phenyl-2-cyclopenten-1-on ${ }^{[7]}$ lieferte das gleiche 2,4-Dinitrophenylhydrazon $\left(\lambda_{\max }=402 \mathrm{~nm}, \log \varepsilon=4.58\right)$. Im ${ }^{1} \mathrm{H}-\mathrm{NMR}$-Spektrum von (11) findet man die isochronen $5-\mathrm{H}_{2}$ als Dublett bei $\tau=6.77$, nur durch $3-\mathrm{H}$ mit $2.0 \mathrm{~Hz}$ aufgespalten.

Für die 1,5-sigmatrope H-Verschiebung von (12) zu (13) gibt es ein Vorbild in einem cyclisch cis-fixierten System ${ }^{[8]}$. (13) geht nicht in (11) über.

Auch (14) öffnete sich in siedendem Cyclohexan zum Viny!keten (15), wie die auf Alkohol-Zusatz verschwindende Gelbfärbung lehrte. Äthyl-vinyläther vermochte (15) in situ unter Bildung von $63 \%$ der Cyclobutanone (16) und (17) $(28: 72)$ abzufangen.

Die Bestrahlung von (14) in $\mathrm{CCl}_{4}$ bei $10^{\circ} \mathrm{C}$ führte zu einer gelben Lösung von (15) mit der typischen Keten-Absorption bei $2097 \mathrm{~cm}^{-1}{ }^{\text {[9] }}$. Die Extinktion von (15) nahm in 3 Tagen bei Raumtemperatur auf die Hälfte $a b$, ohne daß diejenige des Cyclobutenons (14) dafür zunahm. Die Reaktion $(15) \rightarrow(14)$ ist also entweder zu langsam oder es fehlt ihr die thermodynamische Triebkraft.

Eingegangen am 16. April 1975 [Z 233b]

[1] E. F. Jenny u. J. D. Roberts, J. Amer. Chem. Soc. 78, 2005 (1956)

[2] L. I. Smith u. H. H. Hoehn, J. Amer. Chem. Soc. 61. 2619 (1939); 63. 1181 (1941).

[3] J. Druey, E. F. Jenny, K. Schenker u. R. B. Woodward. Helv. Chim Acta 45, 600 (1962); E. W. Neuse u. B. R. Green, Liebigs Ann. Chem. 1974, 1534.

[4] H. Mayr u. R. Huisgen. Angew. Chem. 87, 491 (1975); Angew. Chem. internat. Edit. 14. Nr. 7 (1975).

[5] W. Borsche, Liebigs Ann. Chem. 526, 1 (1936).

[6] Reaktionen der Vinylketene mit nucleophilen Agentien: H. Mayr u. R. Huisgen, noch unveröffentlicht.

[7] H. A. Weidlich u. G. H. Daniels, Ber. Deut. Chem. Ges. 72. 1590 (1939)

[8] P. Schiess u. C. Suter. Helv. Chim. Acta 54, 2636 (1971).

[9] O. L. Chapman u. J. D. Lassila, J. Amer. Chem. Soc. 90. 2449 (1968) beobachteten nach Bestrahlung von Perchlorcyclobutenon bei $-190^{\circ} \mathrm{C}$ die IR-Bande cines Ketens. 